\author{
П. В. ТРУСОВ, А. И. ШВЕЙКИН
}

\title{
МНОГОУРОВНЕВЫЕ МОДЕЛИ МОНО- И ПОЛИКРИСТАЛЛИЧЕСКИХ МАТЕРИАЛОВ: ТЕОРИЯ, АЛГОРИТМЫ, ПРИМЕРЫ ПРИМЕНЕНИЯ
}

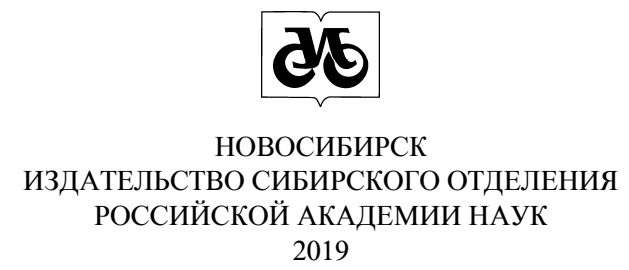




\section{DOI 10.15372/MULTILEVEL2019TPV}

\section{Трусов П. В., Швейкин А. И.}

Многоуровневые модели моно- и поликристаллических материалов: теория, алгоритмы, примеры применения / П. В. Трусов, А. И. Швейкин. - Новосибирск: Издательство СО РАН, 2019. - 605 с.

Работа посвящена последовательному и детальному изложению методологии построения, модификации и реализации многоуровневых моделей для описания деформирования моно- и поликристаллических металлов и сплавов. Модели основаны на введении внутренних переменных и физических теориях упругопластичности и упруговязкопластичности. Приведены основные понятия, аксиомы, законы, соотношения нелинейной механики деформируемого твердого тела. Кратко изложены необходимые для построения рассматриваемых моделей вопросы физики твердого тела. Приведены обзоры публикаций по физическим теориям неупругости и многоуровневым моделям. Представлены формулировки двух- и трехуровневых моделей (главным образом - статистических), учитывающих основные механизмы деформирования: скольжение дислокаций, двойникование, зернограничное скольжение. Значительное внимание уделено проблеме разложения движения, имеющей чрезвычайную важность при построении конститутивных моделей для описания интенсивных неупругих деформаций. Рассмотрены подмодели для описания различных механизмов упрочнения и ротации кристаллитов. Подробно описаны алгоритмы реализации различных модификаций рассматриваемых моделей. Приведены примеры применения разработанных моделей для исследования простого и сложного нагружения макрообразцов. Отдельно рассмотрены результаты использования модифицированной модели для анализа деформирования в режиме сверхпластичности и в переходных режимах. Представлены результаты применения прямой упруговязкопластической модели для исследования деформирования монокристаллического образца, анализируются причины возникновения неоднородности деформирования.

Предлагаемая монография не имеет аналогов в русскоязычной литературе, предназначена для научных сотрудников, специализирующихся в области построения конститутивных моделей (определяющих соотношений) различных материалов, может быть полезна студентам старших курсов физико-механических специальностей и направлений.

Монография подготовлена и издана при финансовой поддержке Российского научного фонда (грант № 17-19-01292)

Рецензенты:

академик РАН Б. Д. Аннин академик РАН В. Е. Панин 


\section{ВВЕДЕНИЕ}

Проблема построения конститутивных моделей, позволяющих описывать поведение материалов в широких диапазонах изменения параметров воздействия (температур, нагрузок, скоростей нагружения и др.), остается одной из наиболее трудных и интересных проблем механики и физики деформируемого твердого тела. Над различными аспектами данной архисложной задачи работают десятки тысяч исследователей - физиков, механиков, металловедов. В последние десятилетия все большее количество научных сотрудников осознают необходимость искать решение проблемы «на стыке» указанных областей знаний; возникла новая область знаний - физическая мезомеханика (В. Е. Панин). В то же время в литературе (по крайней мере - отечественной) практически отсутствуют работы, в которых были бы систематически изложены наиболее важные для построения конститутивных моделей вопросы физики и механики деформируемого твердого тела. Именно это обстоятельство побудило авторов к написанию предлагаемой монографии; в какой мере нам удалось достичь цели комплексного изложения различных аспектов рассматриваемой проблемы - судить Читателю.

Основным объектом исследования в настоящей работе являются металлы и сплавы, точнее - их механическое поведение при различных воздействиях. Конечно, возникает вопрос - почему в наше время стремительно возрастающего интереса к конструкциям из композитных материалов для изучения выбраны именно металлические материалы? Ответ прост: сейчас подавляющая часть конструкций, машин, устройств и т. Д. изготавливаются все-таки из металлических сплавов, и трудно ожидать, чтобы в ближайшие десятилетия ситуация изменилась кардинальным образом. С другой стороны, сплавы представляют собой композитные материалы с более широкими масштабами различных фаз (от миллиметров до нанометров), нежели распространенные конструкционные композиты, с весьма разнообразными физико-химическими связями на атомарном уровне, что позволяет применять подходы, методы, приемы, используемые для построения конститутивных моделей металлических материалов, для разработки моделей композитов. При этом для изготовления изделий из металлических материалов существует чрезвычайно широкий класс технологий, выработанных веками и тысячелетиями человеческой практики, чего в настоящее время нельзя сказать по отношению к композитам.

В последние десятилетия перед механикой деформируемого твердого тела возникли новые вызывающие проблемы, обусловленные актуальными запросами техники и технологий. Прежде всего, данные вызовы связаны с миниатюризацией 
различных устройств и механизмов, используемых в современных изделиях. Появились новые материалы, к числу которых относятся армированные нанотрубками и нановолокнами композиты, так называемые градиентные материалы, получаемые с использованием аддитивных технологий. Необходимость постановки и решения задач исследования поведения конструкций из таких материалов породила резкий рост интереса к, казалось бы, забытым теориям обобщенных континуумов: континууму Коссера, градиентным, микроморфным континуумам. Следует отметить, что большинство исследователей, работающих с моделями обобщенных континуумов, рассматривают материалы с позиций именно сплошной среды.

Все более широкое распространение получают технологии, основанные на интенсивных неупругих деформациях, позволяющие получать изделия с субмикрокристаллической и нанокристаллической структурой металлов и сплавов, обеспечивающей существенное улучшение эксплуатационных характеристик деталей и конструкций. Совершенствование существующих и создание новых технологий обработки материалов невозможно без создания математических моделей анализируемых процессов. «Сердцевиной», ключевым компонентом таких моделей, определяющим их адекватность исследуемым процессам, являются конститутивные модели (определяющие соотношения) для описания поведения рассматриваемых физических тел [Ашихмин и др., 2005]. Большинство существующих моделей для описания поведения металлических материалов, основанных на макрофеноменологическом подходе, не дают возможности анализировать эволюцию микроструктуры, а следовательно - и определять требуемые оптимальные (или рациональные) технологические режимы для получения требуемых микроструктуры и свойств изделий.

В последние десятилетия значительное развитие получили методы (особенно - физические) и «инструментарий» экспериментального исследования поведения материалов при термомеханических воздействиях. Указанные методы позволяют выявлять весьма «тонкие» эффекты в деформируемых металлах и сплавах, связанные с особенностями строения структуры на различных масштабных уровнях. Выявлено существенное влияние на поведение материалов границ образцов и внутренних (межфазных, межзеренных) границ. В связи с этим возникают некоторые вопросы: что же исследуется в экспериментах на образцах - поведение материала или конструкции (образца)? Можно ли считать поведение представительных объемов материала, рассматриваемых в объеме образца и в окрестности его границы, одинаковым? Будут ли указанные представительные объемы описываться идентичными конститутивными моделями? Не требуется ли для решения задач исследования поведения различных деталей (конструкций), по крайней мере, двух типов определяющих соотношений - для материала в объеме и для приграничных областей? Возможно, механика деформируемого твердого тела в части построения конститутивных моделей стоит на пороге коренного изменения парадигмы своего дальнейшего развития - перехода от механики материалов к механике конструкций.

Ответы на эти и другие вопросы, связанные с эффектами, определяемыми микро- и мезоструктурой, по мнению авторов, невозможно получить с помощью классических определяющих соотношений, сформулированных на основе макрофеноменологического, структурно-механического или термодинамического под- 
ходов. Классические модели непригодны и для решения одной из сложнейших проблем современной техники - «конструирования» функциональных материалов (неоднородных материалов, создаваемых под конкретную деталь). Для решения возникающих вопросов и проблем требуются конститутивные модели на «стыке» нелинейной механики деформируемого твердого тела и физики твердого тела. Закономерности поведения конкретных материалов в этом случае необходимо рассматривать на более глубоких масштабных уровнях (мезо-, микро, атомарном), чем в широко распространенных в настоящее время макрофеноменологических конститутивных моделях. Для анализа изготовления и эксплуатации готовых изделий в этом случае понадобится разработка и использование многоуровневых моделей, основанных на физических теориях (упругопластичности, упруговязкопластичности и т. д.).

Условно настоящую работу можно разделить на три части. В первой (глава 1) приведены необходимые сведения из нелинейной механики деформируемого твердого тела (МДТТ), описаны некоторые известные механические эффекты, обнаруженные в макроэкспериментах, но не нашедшие до настоящего времени адекватного теоретического описания. Рассмотрена структура конститутивной модели, основанная на введении внутренних переменных. Представлены основные понятия и определения физики твердого тела (ФТТ), теории дефектов. В последних параграфах главы 1 содержатся обзоры работ по физическим теориям пластичности и многоуровневым моделям; к сожалению, в обзорах фигурируют в основном зарубежные работы, поскольку отечественные публикации по данной тематике встречаются весьма редко.

Глава 2 содержит описание предложенных авторами модификаций физических теорий и многоуровневых моделей. Приведены математические постановки задач на каждом из рассматриваемых уровней (мезо- и макроуровни), даны конкретизация разных типов внутренних переменных и классификация уравнений, входящих в конститутивную модель. Особое внимание уделяется построению моделей мезоуровня (уровня зерна, субзерна), разложению движения на квазитвердое и деформационное, различным формулировкам упругого закона, описанию вязкопластического деформирования, в том числе - в режиме сверхпластичности и в переходных режимах. Наконец, в главе 3 представлены постановки и результаты решения некоторых модельных задач. Рассмотрены результаты исследования различных по сложности процессов нагружения моно- и поликристаллических образцов с разными типами решеток, полученные с использованием модифицированных статистических моделей. Приведены описание процедуры и результаты идентификации и верификации предлагаемых моделей. Анализируется взаимовлияние различных механизмов деформирования (скольжения дислокаций, ротаций кристаллитов, двойникования, зернограничного скольжения). Представлены полученные с применением прямой модели результаты детального исследования деформирования моно- и поликристаллического образцов (возникновение неоднородности, искривление решетки).

Монография предназначена в первую очередь для научных сотрудников, занимающихся построением определяющих соотношений для деформируемых твер- 
дых тел. Полезной она может оказаться для аспирантов и студентов старших курсов механико-математических и физических специальностей.

Над книгой авторы работали свыше 10 лет, добавляя «кирпичики» по мере появления новых интересных публикаций, модификаций собственных моделей, результатов в соответствующие разделы. С этим стилем работы, вероятно, связано различие «весов» разных глав и параграфов. Для облегчения работы с монографией в объемных параграфах введено разделение на подразделы, отраженное в оглавлении. На всех этапах работы над книгой тематика поддерживалась грантами РФФИ и РНФ, за что авторы чрезвычайно благодарны руководству фондов и экспертам.

Авторы выражают свою искреннюю признательность профессору Р. А. Васину, в беседах с которым зародилась и приобрела окончательные формы структура рукописи; надеемся, что многочисленные, длительные обсуждения наиболее важных аспектов предлагаемой работы позволили существенно улучшить его содержание (если это удалось не в полной мере - то вина лежит только на авторах). Авторы весьма благодарны рецензентам академикам РАН Б. Д. Аннину и В. Е. Панину за поддержку тематики, которая не относится к традиционной в сообществе механиков России. Благодарности заслуживают сотрудники кафедры «Математическое моделирование систем и процессов» Пермского национального исследовательского политехнического университета, принимавшие активное участие в работе научного семинара кафедры, создании конкретных моделей, разработавшие и реализовавшие программы для решения рассмотренных в монографии задач, Н. С. Кондратьев, А. Ю. Янц, Э. Р. Шарифуллина, П. С. Волегов, К. В. Остапович, И. Ю. Зубко, В. Н. Ашихмин, Е. С. Макаревич, Т. В. Останина, И. Л. Исупова и другие. 


\section{ОГЛАВЛЕНИЕ}

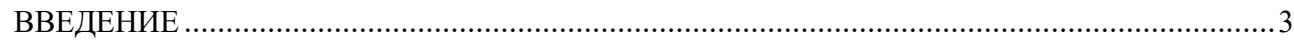

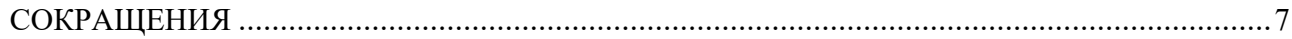

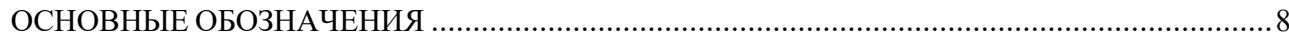

1. УПРУГОПЛАСТИЧЕСКОЕ ДЕФОРМИРОВАНИЕ МЕТАЛЛОВ: МЕХАНИКА,

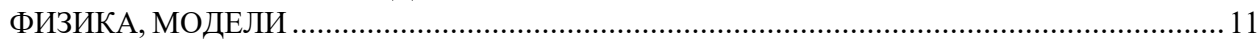

1.1. НЕОБХОДИМЫЕ СВЕДЕНИЯ ИЗ МЕХАНИКИ ДЕФОРМИРУЕМОГО ТВЕРДОГО ТЕЛА ................12

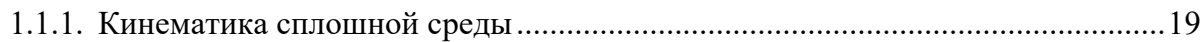

Системы отсчета. Конфигурация. Движение .............................................220

Способы описания движения ..................................................................22

Независимость от выбора системы отсчета ..........................................22

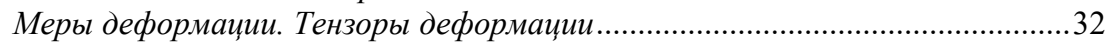

Тензоры приращения и скорости деформации ..............................................4 47

1.1.2. Краткие сведения из динамики сплошной среды ...........................................60

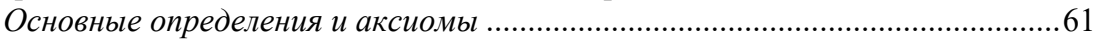

Масса. Аксиомы массы. Закон сохранения массы.

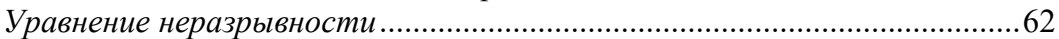

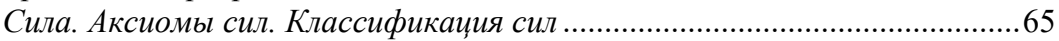

Об уравнениях баланса .................................................................... 70

Количество движения и момент количества движения,

кинетическая энергия, скорость совершения работы ..............................72

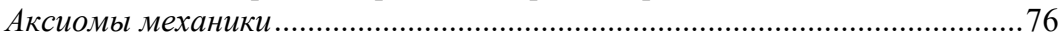

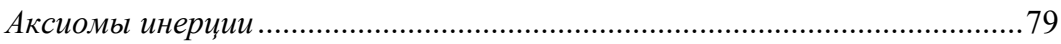

Уравнения движения сплошной среды. Напряжения ....................................82

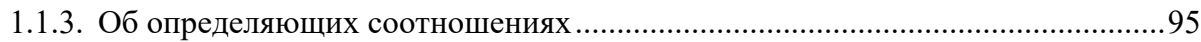

Аксиомы теории определяющих соотношений ……....................................101

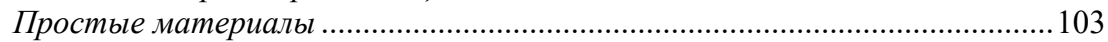

Определяющие соотношения с внутренними переменными ......................... 104

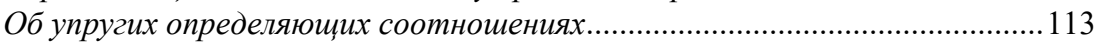

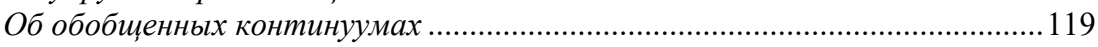

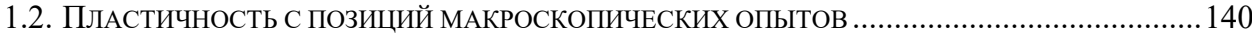

1.3. О ФИЗИЧЕСКИХ МЕХАНИЗМАХ НЕУПРУГОГО ДЕФОРМИРОВАНИЯ ...................................159

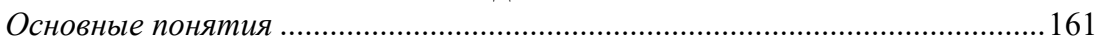

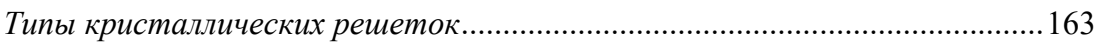

Кристаллографические направления и плоскости ....................................... 166

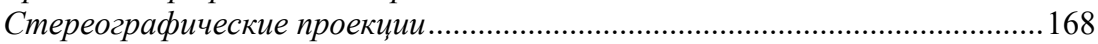

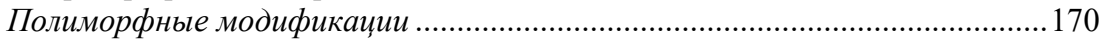

Твердые растворы, химические соединения ……………….........................171

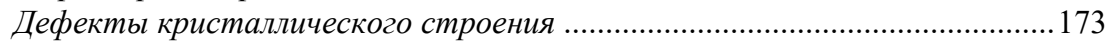

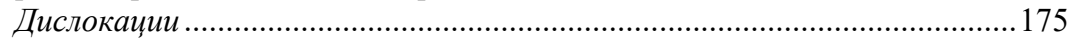


Дислокациионные реакиии ....

Взаимодействия дислокаций с дислокащиями и точечными

дефектами

Дисклинации

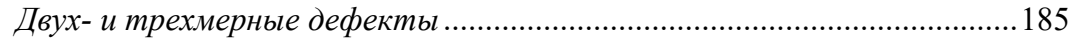

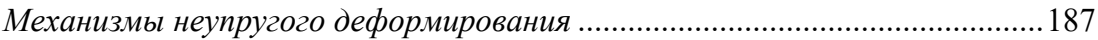

Микроструктура и внутренние переменные .................................................196

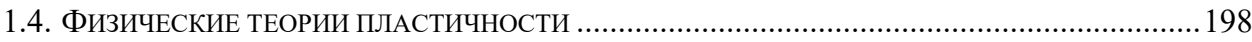

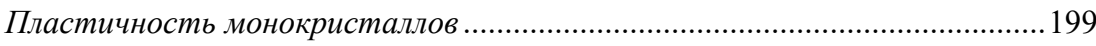

О теориях пластичности для поликристаллов .............................................204

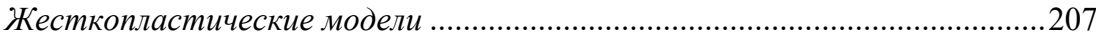

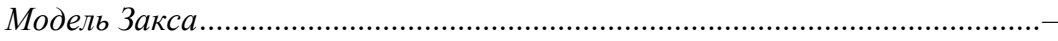

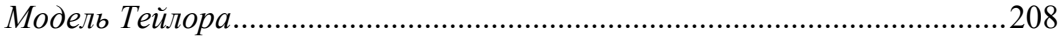

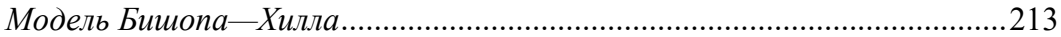

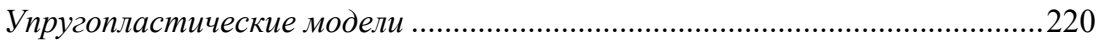

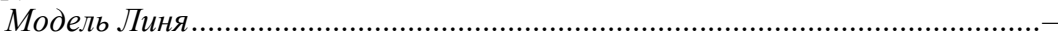

Вязкопластические модели.......................................................................2234

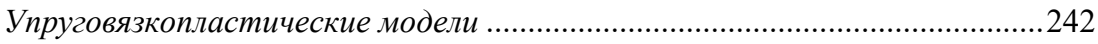

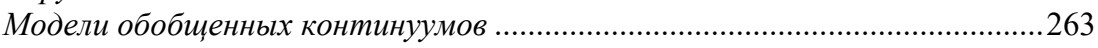

Структурно-аналитическая теория прочности и пластичности...............2273

1.5. МНОГОУРОВНЕВЫЕ МОДЕЛИ ДЛЯ ОПИСАНИЯ ДЕФОРМИРОВАНИЯ И ЭВОЛЮЦИИ

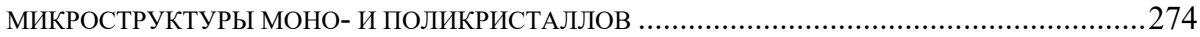

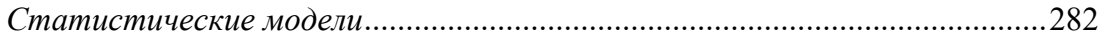

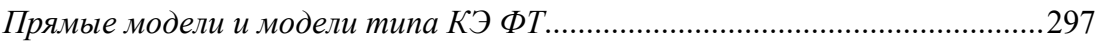

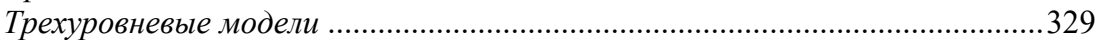

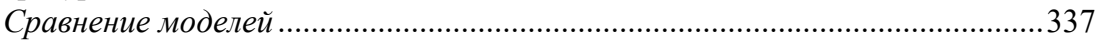

Применение многоуровневых моделей для решения прикладных

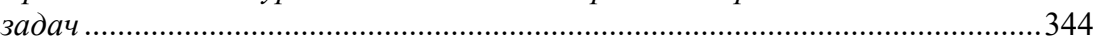

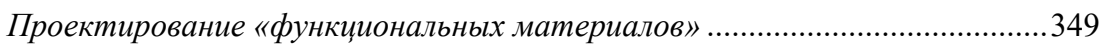

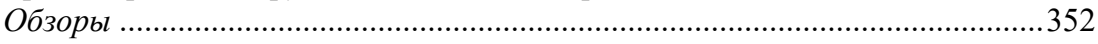

\section{2. МНОГОУРОВНЕВЫЕ МОДЕЛИ ДЛЯ ОПИСАНИЯ НЕУПРУГОГО}

ДЕФОРМИРОВАНИЯ МОНО- И ПОЛИКРИСТАЛЛИЧЕСКИХ МАТЕРИАЛОВ …............358

2.1. О ПОДХОДАХ К ПОСТРОЕНИЮ ГЕОМЕТРИЧЕСКИ НЕЛИНЕЙНЫХ КОНСТИТУТИВНЫХ

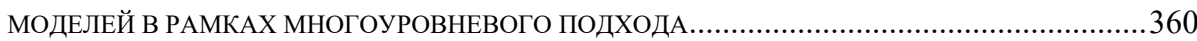

2.2. ОБЩАЯ СТРУКТУРА МНОГОУРОВНЕВЫХ КОНСТИТУТИВНЫХ МОДЕЛЕЙ МАТЕРИАЛА.

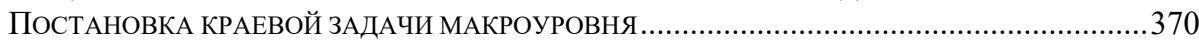

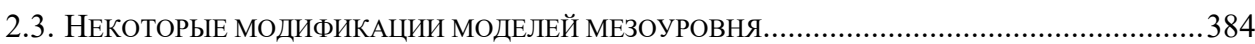

Меры напряженного и деформированного состояния на мезоуровне ..........385

Различные формулировки упругого закона в модели мезоуровня ....................389

Сопоставление моделей: упругое деформирование ........................................394

Сопоставление моделей: неупругое деформирование .....................................397

Описание деформирования кристаллитов за счет скольжения

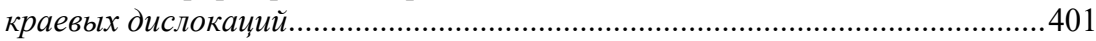

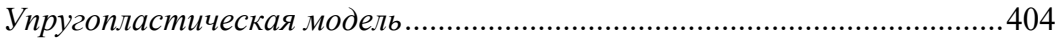

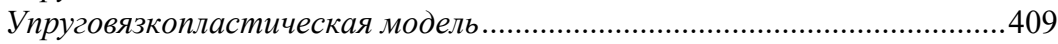

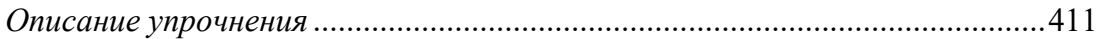

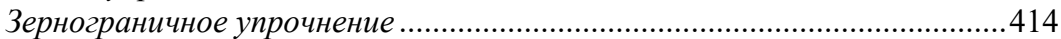

Описание разупрочнения за счет перестройки дефектной структуры 
Модели ротации решетки кристаллитов (соотношения для определения спина подвижной системь координат на мезоуровне) ............426

О возможных направлениях развития моделей мезоуровня.

2.4. ЗАМКНУТАЯ СИСТЕМА УРАВНЕНИЙ ДВУХУРОВНЕВОЙ УПРУГОВЯЗКОПЛАСТИЧЕСКОЙ МОДЕЛИ

2.5. ТРЕХУРОВНЕВАЯ МОДЕЛЬ ДЛЯ ОПИСАНИЯ ДЕФОРМИРОВАНИЯ В РЕЖИМЕ СТРУКТУРНОЙ СВЕРХПЛАСТИЧНОСТИ И ПЕРЕХОДОВ МЕЖДУ РЕЖИМАМИ

Описание результатов экспериментальных исследований

деформирования в режиме сверхпластичности .

Трехуровневая упруговязкопластическая модель для описания сверхпластического деформирования

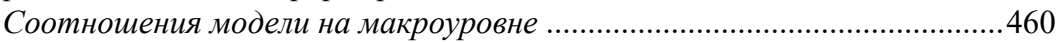

Соотношения модели на мезоуровне ……................................................

Подмодель для описания зернограничного скольжения..............................464

3. ПРИМЕНЕНИЕ МНОГОУРОВНЕВЫХ МОДЕЛЕЙ ДЛЯ ИССЛЕДОВАНИЯ ПОВЕДЕНИЯ МАТЕРИАЛОВ ПРИ ПРОСТЫХ И СЛОЖНЫХ НАГРУЖЕНИЯХ

3.1. ИССЛЕДОВАНИЕ ПОВЕДЕНИЯ ОБРАЗЦОВ ПРИ ПРОСТЫХ НАГРУЖЕНИЯХ .

3.1.1. Результаты моделирования деформирования монокристаллических образцов

3.1.2. Результаты моделирования деформирования поликристаллических образцов 486

3.2. СЛОЖНОЕ НАГРУЖЕНИЕ ПРЕДСТАВИТЕЛЬНОГО МАКРООБЪЕМА

O построении образа прочесса нагружения при больших градиентах перемещений

Примеры исследования деформирования по сложным траекториям при малых градиентах перемещений; сопоставление с экспериментом .....500 Примеры моделирования деформирования по траекториям произвольной сложности при больших градиентах перемещений. .511 Физический смысл неголономной меры деформаџии на макроуровне

3.3. РЕЗУЛЬТАТЫ МОДЕЛИРОВАНИЯ ДЕФОРМИРОВАНИЯ МАКРООБРАЗЦОВ С УЧЕТОМ РЕАЛИЗАЦИИ ЗЕРНОГРАНИЧНОГО СКОЛЬЖЕНИЯ И ПЕРЕХОДА

К РЕЖИМУ СВЕРХПЛАСТИЧНОСТИ .

3.4. ПРЯМОЕ МОДЕЛИРОВАНИЕ ДЕФОРМИРОВАНИЯ ПРЕДСТАВИТЕЛЬНЫХ ОБЪЕМОВ МОНО- И ПОЛИКРИСТАЛЛОВ

Моделирование деформирования монокристалла ….................................553

Моделирование деформирования поликристалла....................................543

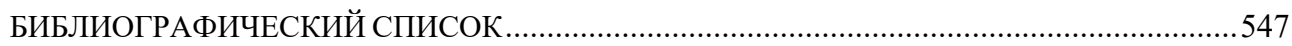

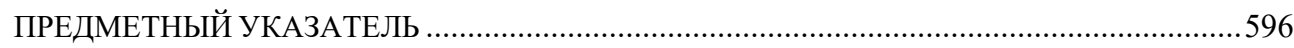


Научное издание

Трусов Петр Валентинович

Швейкин Алексей Игоревич

\section{МНОГОУРОВНЕВЫЕ МОДЕЛИ МОНО- \\ И ПОЛИКРИСТАЛЛИЧЕСКИХ МАТЕРИАЛОВ: ТЕОРИЯ, АЛГОРИТМЫ, ПРИМЕРЫ ПРИМЕНЕНИЯ}

Редактор Н. А. Лившии

Корректор Н. В. Счастнева

Технический редактор $H$. В. Бутакова

Подписано в печать 06.12.2019. Формат $70 \times 100$ 1/16.

Усл. печ. л. 49,16. Уч-изд. л. 49,52. Тираж 300 экз. Заказ № 241

Издательство СО РАН

630090 Новосибирск, Морской просп., 2

E-mail: psb@sibran.ru

тел. (383) 330-80-50

Отпечатано в Издательстве СО РАН

Интернет-магазин Издательства СО РАН

http://www.sibran.ru 